\title{
PATRIMONIO GASTRONÓMICO Y MUJERES OTOMÍES EN EL ESTADO DE MÉXICO, MÉXICO: UN PROBLEMA DE ROLES DE GÉNERO
}

\section{Gastronomic heritage and Otomi women in the State of Mexico, Mexico: a problem of gender roles}

\section{Arlen Sánchez Valdés}

Profesor de Tiempo Completo de la Facultad de Turismo y Gastronomía, Universidad Autónoma del Estado de México (UAEM).

Correo-e: arlensanchezvaldes@gmail.com ORCID: 0000-0001-5241-4123,

\section{Alejandro Delgado-Cruz}

Profesor de Tiempo Completo de la Facultad de Turismo y Gastronomía, Universidad Autónoma del

Estado de México (UAEM).

Correo-e: adelgadoc@uaemex.mx

ORCID: 0000-0002-9135-9304

\author{
Bibiana Gónzalez Acosta \\ Licenciada en Gastronomía por la Facultad de Turismo \\ y Gastronomía, Universidad Autónoma del Estado de \\ México (UAEM). Correo-e: bga_1202@hotmail.com \\ ORCID: 0000-0003-0882-4031
}

Recibido: 1/7/2020 • Aprobado: 28/7/2020

Cómo citar: Sánchez Valdés, A., Delgado-Cruz, A., \& González Acosta, B. (2020). Patrimonio gastronómico y mujeres otomíes en el Estado de México, México: un problema de roles de género. Ciencia y Sociedad, 45(3), 85-96. Doi: https://doi.org/10.22206/ cys.2020.v45i3.pp85-96

\section{Resumen}

El objetivo del artículo es analizar el papel de las mujeres indígenas de la comunidad otomí de San Pablo Autopan, en el Estado de México, México; en la preservación del patrimonio gastronómico. A través de un estudio fenomenológico, se logra abordar la compleja relación entre los roles de género y el patrimonio gastronómico. Los resultados muestran que, a pesar de la conciencia comunitaria, el patrimonio gastronómico está en riesgo de perderse debido a los procesos de globalización, la contaminación ambiental y el cambio de roles de género en las nuevas generaciones junto con las presiones económicas. Se concluye que la preservación del patrimonio gastronómico debería ser una actividad comunitaria compartida entre hombres y mujeres en equidad de circunstancias.

Palabras clave: patrimonio gastronómico; mujeres indígenas; roles de género; rol sexual.

\begin{abstract}
The aim of the paper is to analyze the role of indigenous women from the Otomí community of San Pablo Autopan, in the State of Mexico, Mexico; in the conservation of the gastronomic heritage. Through a phenomenological study, it is possible to address the complex relationship between gender roles and gastronomic heritage. The results indicate that despite community awareness, this heritage is at risk of being lost due to the processes of globalization, environmental contamination and change of gender roles in new generations along with economic crises. It is concluded that the conservation of the gastronomic heritage should be a community activity shared by men and women in equity circumstances.
\end{abstract}

Key words: Gastronomic heritage; indigenous women; gender roles, sexual role. 


\section{Introducción}

Los alimentos y bebidas son vistos como símbolos de cultura e identidad grupal. Los platillos e ingredientes comunican algo significativo sobre las personas, tanto al interior como al exterior de los grupos, a menudo en procesos socioculturales (Gatley, 2016). Este tipo de visión, ha provocado un creciente interés en la academia por el pasado y presente de los alimentos, así como por las prácticas asociadas con su producción, consumo y preservación, donde el rol de género condiciona esta actividad, no solo en México, sino en otras partes del mundo (Hryciuk, 2019; González, 2019; Matta, 2019).

En este tenor, la gastronomía indígena está capturando la atención de la ciencia. Los estudios de este campo se basan en los análisis de las expresiones culinarias tradicionales, la dinámica cultural que viven las comunidades y cómo esta afecta directa o indirectamente en los platillos (Barrionuevo, Bernat y Velarde, 2019; Gatley, 2016; González, 2019; Sánchez y Goldsmith, 2000). Sin embargo, hacen falta investigaciones que develen el papel de las mujeres indígenas en la preservación de dicho patrimonio, siendo que día con día aumenta la presión por cambiar los hábitos de consumo debido a los procesos de globalización, la contaminación ambiental y el cambio de roles de género en las nuevas generaciones, que junto con las crisis económicas han obligado a las mujeres a cambiar sus labores del hogar por otras más redituables (Hryciuk, 2019; Matta, 2019; Pederzini, 2008).

Tomando como base los roles de género y la socialización (Ridgeway, 2011), se busca discutir cómo el patrimonio gastronómico de la comunidad de San Pablo Autopan en el Estado de México, enfrenta un problema de estereotipo de roles de género, que pone en riesgo su prevalencia. A pesar de observar una organización social y familiar, se identifica que los lazos sociales se conservan gracias a una fuerte estructura basada en tradiciones y fiestas, que mantienen a los otomíes unidos como un grupo con identidad común (Barrientos, 2004). La comunidad objeto de este estudio, atraviesa un momento complicado ya que, por razones económicas y sociales, gran parte de sus miembros está dejando de lado su gastronomía y su idioma. Por tal motivo, el objetivo es analizar el papel de las mujeres otomíes pertenecientes a la comunidad de San Pablo Autopan, en la preservación del patrimonio gastronómico.

Este documento se estructura de la siguiente manera. Primero, se presenta el tema del patrimonio gastronómico en las comunidades indígenas y se abordan las concepciones en torno a los roles de género y socialización. Posteriormente, en la metodología, se expone el enfoque, diseńo y alcance del estudio. Se continua con el análisis y discusión de los resultados para después desprender las conclusiones y futuras líneas de investigación.

\section{La gastronomía como patrimonio cultural y su valor comunitario}

En México, al igual que en otros países de América Latina, la gastronomía es parte del patrimonio cultural, forjador de la identidad colectiva y base de afectos, costumbres y reciprocidad, donde los rituales alimenticios son factores de cooperación y cohesión social (López, Juárez y Medina, 2017). Desde el siglo pasado, las transformaciones culturales y sociales asociadas a la gastronomía han despertado el interés de varias investigaciones en el área de la antropología, demostrando que en el ámbito de la comida se presentan oportunidades excepcionales para indagar más sobre ellas (Mintz, 1999; López et al., 2017; Weismantel, 1991).

De igual manera, Rodríguez, Chávez, Thomé y Miranda (2017) conceden la idea de que los alimentos pueden tener un significado diferente dependiendo de la forma y contexto de su elaboración, reconfigurando sus usos y apropiaciones; el mismo producto podría ser a la vez un símbolo de identidad y un recurso económico. Por su parte, 
Hernández (2016) analiza las interpretaciones de la comida desde la etapa de la producción hasta el consumo, indicando que los alimentos dentro de la vida de los pueblos indígenas están relacionados con las concepciones respecto a lo sagrado para propiciar la armonía y el bienestar, tanto a nivel individual como grupal.

La Food and Agriculture Organization (FAO) ha mostrado la relevancia del estudio de la gastronomía de los pueblos indígenas, recolectando una explicación de su cosmovisión, su memoria de la comida, siglos de costumbres, de tradición, de conocimientos, de abundancia y de escasez; enfocándose en el papel de las raíces de la alimentación indígena, sus ingredientes y sus formas de producción, como aspectos esenciales en la construcción de la identidad y patrimonio de estos pueblos (FAO, 2015). Sin embargo, más allá del valor patrimonial de los alimentos, su consumo está determinado por: a) componentes biológicos (la palatabilidad y las sensaciones de hambre o saciedad), b) económicos (el costo, los ingresos y la disponibilidad en el mercado), c) físicos (el acceso, la zona geográfica, la educación, el estilo de vida o las capacidades personales - por ejemplo, para cocinar- y el tiempo disponible) y d) sociales (la cultura, la familia, y los patrones de alimentación) (Dip, 2014). De alguna forma, se incorporan los aspectos de la vida moderna a los hábitos alimenticios, marcando las pautas para nuevos estudios en comunidades que enfrentan procesos de transformación, en especial de cambio de roles en las mujeres guardianas del patrimonio gastronómico (Hryciuk, 2019; Matta, 2019).

Por otro lado, Entrena (2008) y De Suremain (2017) evidencian que el patrimonio gastronómico se ve alterado en sus raíces cuando los hábitos alimentarios cambian por la pérdida de alguno de sus elementos o la inclusión de otros, mismos que impactan en la prevalencia del conjunto de significados, al tiempo de afectar la identidad colectiva y con ello, propiciar su reconfiguración. De tal forma, que la realidad de los hábitos alimentarios cambia en el momento en que los escenarios de convivencia social se expanden. O bien, tal como lo afirma Ochoa (2013), el papel de los roles de género adoptados para la preparación de alimentos marca las pautas de su evolución. En consecuencia, la herencia de los alimentos tradicionales todavía permanece en los pueblos indígenas, aunque parte de esta se ha perdido ante la llegada de productos exógenos y los cambios de roles sociales de sus miembros.

En suma, la diferenciación social, los roles de género y la búsqueda de la comunidad por mantener su patrimonio e identidad son elementos sustanciales y fortuitos. Empero, esto no significa cerrarse al contacto y la apropiación de elementos exógenos del mundo moderno y globalizado para reconfigurar sus pautas socioculturales establecidas, teniendo claro el significado de estos cambios y conservando la esencia del patrimonio gastronómico local.

\section{Roles de género y socialización: la realidad de las mujeres indígenas}

La teoría de la congruencia de rol de Eagly (1987), permite entender cómo las asociaciones expresan expectativas compartidas sobre la conducta de las personas con base en su sexo socialmente identificado, es decir, expresan roles de género dando origen a procesos de discriminación en gran medida, debido a que los roles incluyen normas de conductas típicas y prescritas para cada género. Así, los roles se basan en las diferentes posiciones que ocupan mujeres y hombres en la división sexual del trabajo o sesgo de correspondencia (Eagly, Wood y Johannesen, 2004).

Por su parte, Ridgeway (2011) busca explicar cómo la cultura y el contexto histórico propician que el género y otras formas de inequidad puedan prevalecer. Concibiendo al género como un sistema de prácticas sociales donde a través de diferencias sexuales se establecen etiquetas. Siendo que desde 
el momento en que esta asignación se realiza, la vida de esa persona se ve influenciada y dirigida por formas de actuar, expectativas y obligaciones impuestas por este rol (Ortiz, 2015). Desde una perspectiva de género, la división sexual del trabajo y la asignación de roles para hombres y mujeres, tiene como consecuencia crear disparidades sociales y culturales (Aguilar, 2016).

Respecto al vínculo entre gastronomía y roles de género en grupos indígenas, los significados de socialización que se detonan son más profundos (Ochoa, 2013). Al ser comunidades con una riqueza cultural y tener un respeto celoso de la misma, la socialización como proceso les permite aprender e interiorizar toda una serie de normas, valores y formas de percibir la realidad, que los dotarán de las capacidades necesarias para desenvolverse satisfactoriamente en la interacción social (Aguilar, 2016). Así, las mujeres desde su nacimiento están determinadas a dedicarse al hogar y, en particular, al cuidado de los infantes y la cocina. Sin embargo, la socialización se debe entender como un proceso temporal y evolutivo (Ortiz, 2015).
Desde una perspectiva de género, el rol está basado en una evolución de la división sexual del trabajo. En las comunidades indígenas, este rol cambiará durante la vida de las personas y marcará las pautas de desarrollo, así como la preservación del patrimonio gastronómico. Por eso, cada vez es más recurrente que muchas mujeres jóvenes rechacen el rol impuesto por patrones sociales que las obligan a limitarse a la cocina y las aleja de espacios de mayor retribución económica y reconocimiento social.

\section{Metodología}

Los otomíes son un pueblo originario con gran presencia desde la zona centro del país y hasta el Golfo de México, incluyendo las entidades de Hidalgo, Guanajuato, Querétaro, Puebla y Veracruz. Siendo una de las etnias más relevantes por el número de sus representantes y esencia cultural (Barrientos, 2004; Montoya y Sandoval, 2013). Así, el trabajo de campo se llevó a cabo durante los meses de agosto a octubre del año 2019 en la comunidad de San Pablo Autopan, perteneciente al municipio de Toluca, Estado de México, México (figura 1).
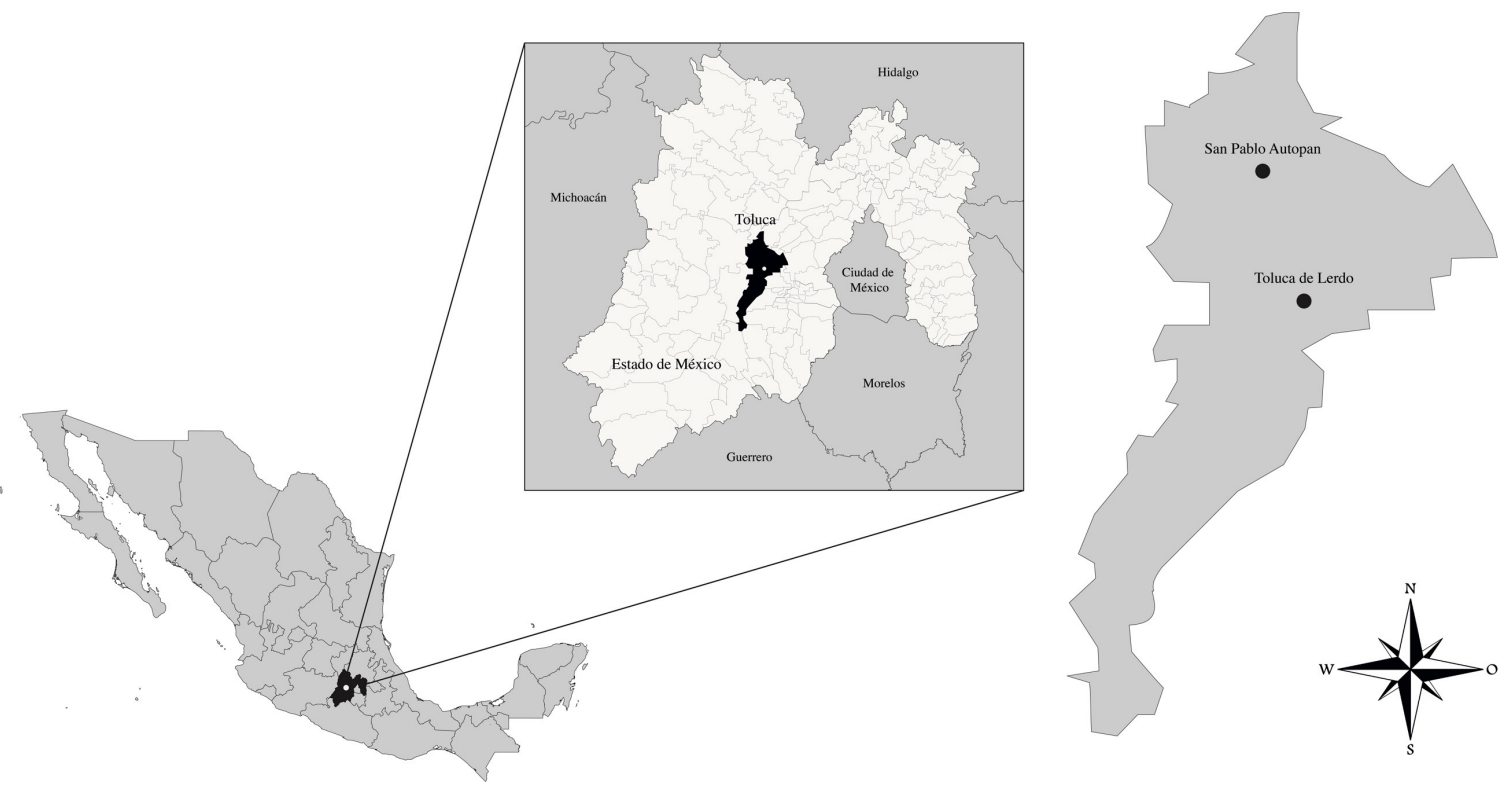

Figura 1. Ubicación de San Pablo Autopan, Estado de México

Fuente: elaboración propia.

88 | Ciencia y Sociedad 2020; 45(3, julio-septiembre): Mirada desde lo particular • 85-96 
La investigación tuvo un enfoque cualitativo, diseño fenomenológico y alcance descriptivo (Creswell, 2017) al centrarse en los aspectos subjetivos y emanados de las experiencias compartidas de las mujeres otomíes en torno a la gastronomía y el rol de género. En este sentido, se trabajó con mujeres integrantes de dicha comunidad. Primero, se hizo un reconocimiento previo de la comunidad a fin de identificar que las informantes contaran con las características requeridas: a) mujeres adultas que cocinan o acostumbraban cocinar, b) originarias de la comunidad y c) que actualmente residieran en la zona de estudio. Después, se invitó a quienes cumplieron con los criterios, garantizándoles el uso académico de los datos, así como de su confidencialidad y anonimato.

En cuanto a la técnica de recolección de datos, se empleó la entrevista a profundidad con apoyo de un guion semiestructurado. Esta actividad se llevó en los hogares de las mujeres. El propósito de las entrevistas fue entender a las mujeres indígenas, su rol y su relación con el patrimonio gastronómico. Evidenciando la lucha por la preservación de los roles de género, pero también surgiendo temas relevantes para la investigación como los daños ecológicos que han impactado en la disponibilidad de materias primas para cocinar y cómo las mujeres deben salir a trabajar para sobrevivir a las presiones económicas que enfrentan.

Para determinar el número de entrevistas, inicialmente se consideraron las recomendaciones de Hernández y Mendoza (2019) para estudios fenomenológicos, indicando un número idóneo de diez personas; no obstante, la sobresaturación de categorías figuró como el criterio para decidir el número de informantes. En este caso fueron 12 mujeres abordadas, puesto que a partir de las entrevistas número 10 , se dejó de ofrecer datos y contenidos novedosos o divergentes respecto al objeto de estudio y la explicación teórica (Díaz, Torruco, Martínez y Varela, 2013).
Por otro lado, se empleó el análisis de contenido para el tratamiento de datos, lo que permitió interpretar el discurso de las informantes con detalle y profundidad y, con ello, develar significados. Para llevar a cabo esta técnica fue necesario transcribir las expresiones orales, para posteriormente hacer la codificación abierta de palabras, frases y párrafos que exteriorizaban aspectos relevantes en torno al patrimonio gastronómico y los roles de género. Una vez realizado esto, se procedió a la codificación axial donde se relacionaron y agruparon los códigos, a la par de definir las subcategorías según su concurrencia y peso reflejado en las expresiones de las informantes. En el último análisis, fue necesario jerarquizar las subcategorías y categorías y brindar un significado de sus asociaciones con el objeto de estudio.

Cabe mencionar que los datos fueron tratados con apoyo de ATLAS.ti, un programa de análisis cualitativo asistido por computadora (QDA, por sus siglas en inglés) que permitió (a) asociar códigos o etiquetas con fragmentos de texto, (b) buscar códigos de patrones y, (c) clasificar y jerarquizar las categorías resultantes (Hwang, 2007).

\section{Resultados}

La primera etapa consistió en un análisis exploratorio mediante la técnica de nube de palabras, que ayudó a identificar los códigos más relevantes, generando una guía rápida de los contenidos. En la figura 2, se puede observar que los términos más concurrentes son aquellos que hacen alusión a los alimentos tradicionales, la identidad y los cambios en la cocina de la comunidad de San Pablo Autopan.

Por su parte, los ingredientes utilizados son las calabazas, el maíz, los frijoles, el chile, los quelites, el arroz, el pescado, los acociles, las tortillas, el mole y los tamales, entre otros, evidenciando una dieta lacustre y basada en la milpa, dada su ubicación geográfica cercana al bordo de San Jerónimo 
(manto acuífero) y su tradición del cultivo del maíz junto con otras hortalizas (figura 2).

En cuanto al tema del género, se puede apreciar que las expresiones relativas a las mujeres: "hijas", "mamá" y "abuelita" son recurrentes, debido a que las actividades de la cocina y el resguardo del patrimonio alimentario se reservan para ellas, lo que refuerza el rol de género que les ha sido asignado; mientras que la palabra "comunidad" refleja la parte social que aún prevalece (figura 2).

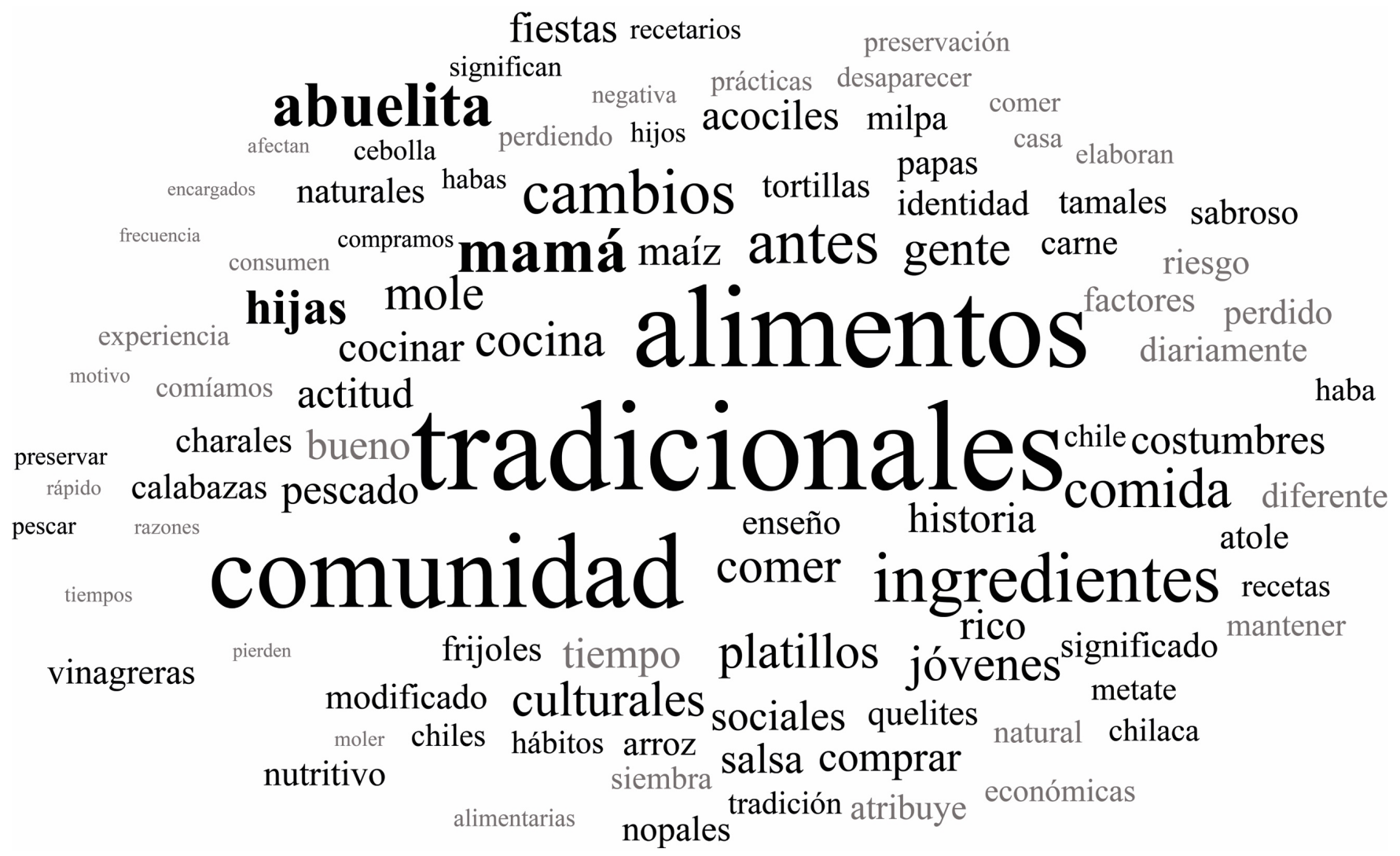

Figura 2. Nube de palabras

Fuente: elaboración propia.

En cuanto al patrimonio gastronómico, las mujeres mayores de la comunidad son las encargadas de preservar los conocimientos sobre la tierra, la siembra, la cosecha, las prácticas culinarias y el resto de los saberes transmitidos de generación en generación de forma oral o demostrativa: "Me enseñaba mi mamá, nos hacían unos platillos (...), veíamos cómo lo hacía y (...) ya le íbamos aprendiendo" (Entrevistada 1). Lamentablemente, todo este conjunto de saberes y conocimientos gastronómicos a la fecha no se han logrado documentar en algún recetario, puesto que sus portadoras no saben escribir, o tienen una edad muy avanzada.

Por otro lado, la cocina ha sido tradicionalmente un escenario para las mujeres, contrariamente, en los últimos años, se ha observado el interés por parte de los varones más jóvenes por integrarse en esta actividad: "los jóvenes hombres de la comunidad ahora disfrutan cocinar más que las mujeres" (Entrevistada 3), evidenciando que los roles de género están visualizándose desde una óptica 
renovada y menos cerrada frente a las normativas sociales sobre el papel que tiene un hombre y una mujer, al contrario, emancipan el juicio, la creatividad y la responsabilidad para seguir preservando la cocina otomí.

Los desarrollos industriales de la región, las constantes crisis económicas, así como la improductividad de varias parcelas afectadas por los contaminantes, han limitado la economía agrícola de auto-consumo. Al mismo tiempo se propicia la migración laboral de las mujeres y con ello, cambiar los roles que por generaciones se les habían asignado: cuidado de la familia, la casa y la cocina. Así, la vida cotidiana de la mujer otomí ahora se divide en tareas del hogar y el campo, esta última actividad como fuente de ingresos adicionales.

En este sentido, las mujeres empiezan a salir a trabajar por dos razones principales. En la primera, se observa que muchas de ellas tuvieron la oportunidad de tener una educación escolarizada básica, por lo que han decidido salir a trabajar en comunidades cercanas, donde se han instalado complejos industriales que han favorecido el empleo: "aquí en San Pablo, la mayoría de las mujeres trabajan ya que tienen que ayudar económicamente a su pareja" (Entrevistada 1). La diversificación de las actividades de las amas de casa resultó en la reducción de los tiempos dedicados a la preparación de los alimentos tradicionales, por lo que su elaboración cayó en desuso y algunas de las técnicas culinarias que se precisaban para su elaboración, se fueron abandonando. Asimismo, la introducción de tecnologías en la cocina, les permite tener más tiempo para otras actividades: "las mujeres que todavía pueden hacerlo siembran, cosechan y cocinan, ahora ya hay estufas y licuadoras, antes todo se hacía a mano" (Entrevistada 5).

En cuanto a la segunda razón, esta gira en torno a la contaminación del bordo, ya que desde hace más de cuatro décadas se empezó a limitar la disponibi- lidad de productos de consumo y del agua de riego para la milpa, encareciendo la vida y ejerciendo presión económica en la familia, sin que esto significará abandonar las labores domésticas. En este aspecto, se habla de que antes los productos que se obtenían del bordo para consumo, también servían como trueque con comunidades cercanas, por lo que el dinero no era un recurso tan necesario como es en la actualidad: "los peces y ranas se cambiaban por ollas o, productos de limpieza y aseo personal" (Entrevistada 2).

A pesar de los problemas de contaminación de los recursos acuícolas y la pérdida de terrenos cultivables frente a la invasión de la mancha urbana, la comunidad aún trata de mantener una relación orgánica con el ambiente a través del uso y aprovechamiento responsable de los recursos prevalecientes. Sin embargo, cada día es más complicada y costosa la obtención la materia prima para las recetas tradicionales: "antiguamente, en el bordo había ranas, peces blancos, charales, acociles; ahora todo lo conseguimos en la tienda y no sabemos de dónde lo traen" (Entrevistada 4), “(...) ahora todo es más caro, es más difícil comprar (Entrevistada 3).

Respecto al valor comunitario, las entrevistadas externaron en repetidas ocasiones que al perderse los bordos, también se desapareció la pesca como un hábito colectivo que facilitaba la convivencia entre los miembros con otras comunidades, especialmente por actividades económicas: "las señoras que sabían pescar (...) iban a Santiaguito (...) que pertenece (...) al municipio de Almoloya Bueno, pues porque allá elaboran cazuelas y ollas jarritos platitos (...) para hacer un trueque un cambio" (Entrevistada 1).

Sin duda, una de las principales modificaciones en la dieta es resultado de la pérdida del bordo, fuente primaria de recursos lacustres, pues cuando el pescado dejó de estar al alcance de la población, se integraron nuevos alimentos: "antes no había que 
atún o jamón, antes eran más quelites y pescado, pescado seco, verdolagas" (Entrevistada 4). Aunado con esto, se hace referencia a que los ingredientes nativos son más baratos, pero al dejar la actividad del cultivo, solo queda la opción de comprarlos en el mercado semanal o la central de abastos: "ya no tenemos milpa y ya no cosechamos, ahora hay que comprar lo más barato y lo más cerca" (Entrevistada 1), "ahora todo lo compramos en el mercado de los martes o en la tienda" (Entrevistada 4).

\section{Discusión}

La cultura otomí tiene elementos identitarios que provienen de la época precolombina y la colonia y que se encuentran adaptados al actual desarrollo capitalista, que son perceptibles en sus actividades económicas, forma de organización familiar, religiosidad y gastronomía, entre otros (Barrientos, 2004; Montoya y Sandoval, 2013). En este sentido, el patrimonio gastronómico y los roles de género presentados por la comunidad otomí de San Pablo Autopan, poco a poco han ido cambiando como resultado de la urbanización, al perder alguno de sus componentes o integrar uno nuevo (De Suremain, 2017; Entrena, 2008). En particular, los hallazgos fueron similares a los de Ochoa (2013), donde ya se había evidenciado que el papel de los individuos está marcado por roles de género asumidos en la preparación de alimentos.

Desde la teoría de la congruencia de rol de Eagly (1987), la comunidad estudiada expresa una serie de expectativas y normas de conducta sobre las mujeres y su vínculo con la cocina con una evidente división sexual del trabajo. Sin embargo, se develó que las mujeres adquieren nuevas responsabilidades y roles que antiguamente eran exclusivos de los hombres, como respuesta a la adaptación de los cambios sociales, económicos y ambientales. Mientras que los varones de la comunidad no necesariamente están adquiriendo los roles que solo eran destinados a las mujeres, creando un desequilibrio social. Sin embargo, se reconoce que los hombres más jóvenes están presentando un interés en la cocina, lo cual pudiera incidir en la preservación del patrimonio gastronómico, aunque faltan investigaciones que soporten este hecho. Además, una limitante es la documentación del cúmulo de saberes de la cocina otomí, ya que hasta el momento todo se transmitido de manera oral y demostrativa por parte de las cocineras más experimentadas (mamás $\mathrm{y}$ abuelas) hacia sus hijas, principalmente.

Los roles de género y la socialización permiten entender por qué las mujeres indígenas han sido las encargadas de la cocina, desde la compra diaria de alimentos — de manera principal- en mercados locales, hasta la producción de platillos y la sobremesa. Se aprecia que antes, en la zona, existía el comercio y se mantenía un sistema de trueque con las comunidades vecinas a través de sus mercados (González, 2019), en donde este rol comercial también era asumido de las mujeres al cambiar pescado, tortillas u otras hortalizas con productos oriundos de comunidades cercanas. Asimismo, la mujer ha sido responsable de manejar los recursos naturales en el ámbito doméstico y de preparar la comida para su familia, independientemente de cualquier dificultad ecológica o económica (Edmunds, Slayters y Rocheleau, 1995).

$\mathrm{Al}$ igual que en otros estudios, este trabajo muestra que las mujeres otomís dedican más tiempo y esfuerzos en la cocina que aquellas que residen en la ciudad (Pederzini, 2008), no solo por la carencia de servicios e infraestructura, sino también por las jornadas que cumplen tanto en el campo como en otras actividades orientadas a generar ingresos adicionales (Sánchez y Goldsmith, 2000). En conjunto, los factores sociales, económicos y ambientales han obligado a las mujeres a desempeñar otros roles, reflejando los complejos escenarios que estarían enfrentando las comunidades indígenas en un futuro inmediato. 


\section{Conclusiones}

El patrimonio gastronómico de San Pablo Autopan -al igual que otros pueblos indígenas del centro de México — tiene como base la milpa (maíz, frijol calabaza y chile) y los recursos lacustres (charales, acociles, algas, entre otros). Empero, la preservación del patrimonio gastronómico está en riesgo de perderse principalmente por dos razones: el cambio en los roles de género y la contaminación del bordo, antigua fuente de abasto de la materia prima para cocinar.

En cuanto a los roles de género, se debe entender que las mujeres de la comunidad durante décadas han desempañado varios papeles vinculados con la producción de los alimentos, no solo como cocineras, sino también desde las actividades de siembra y cosecha. Asimismo, las mujeres se involucran en las formas de consumo, pues son quienes determinan la selección de los alimentos con base en los escenarios sociales, económicos y culturales. Sin embargo, las nuevas generaciones de mujeres han asumido otros roles sociales y económicamente más redituables, por lo que solo se reconoce a las personas adultas como guardianas del patrimonio que no han logrado documentar.

Hasta hace algún tiempo, se asumía que las tradiciones -incluyendo la gastronomía- se heredarían de manera natural de generación en generación, pero los cambios ambientales, sociales y económicos conceptualizan escenarios disruptivos alejados de experiencias pasadas. En particular, la contaminación del bordo provocada por la introducción del drenaje, fue el primer parteaguas para la reconfiguración gastronómica, lo cual se dio décadas antes de iniciar los procesos de cambios de roles en las mujeres.

Desde que nacen, a las mujeres de la comunidad se les asigna un rol de género, mismo que no es compartido y que implica la adquisición incons- ciente de una responsabilidad social y cultural, aunque la preservación del patrimonio gastronómico debería ser una actividad compartida entre hombres y mujeres en equidad de circunstancias. No obstante, los resultados de esta investigación no dejan claro si las nuevas generaciones están dispuestas a asumir la responsabilidad de preservar la identidad cultural a través de su gastronomía, sin importar su género.

A pesar de que la población de San Pablo Autopan posee un gran arraigo a la cocina y productos tradicionales otomíes, la contaminación ambiental, el cambio de estilo de vida y la diversificación de los roles de las mujeres ponen en riesgo el futuro gastronómico. Por tanto, se consideran líneas de investigación las relacionadas con las transformaciones socioculturales, económicas y ambientales, y su impacto en la preservación del patrimonio gastronómico. Otros estudios podrían abordar el rescate de los conocimientos culinarios a través de su documentación en recetarios, describiendo las formas de preparación, orígenes y los momentos en que los platillos son consumidos. Igualmente, son una oportunidad los trabajos que involucren iniciativas, emprendimientos y políticas para promover la gastronomía indígena mediante el apoyo público y privado.

Respecto a las limitaciones del trabajo, se asume la posición cualitativa al centrarse en los aspectos subjetivos y particulares que se engloban en el fenómeno. Por tanto, los resultados no se pueden generalizar para dar explicación a realidades semejantes. Otra limitante fue el uso único de la entrevista para recolectar los datos, recomendando que se pueda optar por otras alternativas metodológicas, por ejemplo, la observación participante y no participante, así como las técnicas narrativas para dilucidar más sobre la transición de los roles de género y el patrimonio gastronómico en el contexto de los pueblos indígenas. 


\section{Referencias}

Aguilar, N. S. (2016). Distribución del trabajo, según roles de género. El caso del Centro de Desarrollo y Educación Especial El Ángel. (Tesis de Maestría). Ecuador, Universidad Técnica de Ambato.

Barrientos, G. (2004). Otomíes del Estado de México. México, Comisión Nacional para el Desarrollo de los Pueblos Indígenas (CDI).

Barrionuevo, C.A., Bernat, E.E. y Velarde, I.J. (2019). We recovered food heritage, and then? Value enhancement and promotion of local agri-food products in Argentina and Spain. British Food Journal, 121(12), 3168-3180. Doi: https://doi. org/10.1108/BFJ-10-2018-0711

Creswell, J. W. (2017). Qualitative inquiry and research design: Choosing among five approaches ( $5^{\mathrm{a}}$ ed.). Londres, Sage Publications.

De Suremain, C. E. (2017). Cuando la alimentación se hace patrimonio. Rutas gastronómicas, globalización y desarrollo local (México). Trace, (72), 165-181. Recueprado de https:/doi. org/10.22134/trace.72.2017.114

Díaz, L., Torruco, U., Martínez M. y Varela, M. (2013). La entrevista, recurso flexible y dinámico. Investigación en Educación Médica, 2(7), 162-167.

Dip, G. (2014). La alimentación: Factores determinantes en su elección. Revista de Divulgación Cientifica, (1). Doi: http://experticia.fca. uncu.edu.ar/numeros-anteriores/n-1-2014

Eagly, A. H. (1987). Sex differences in social behavior: A social-role interpretation. Hillsdale: Lawrence Erlbaum Associates.

Eagly, A. H., Wood, W. y Johannesen, M. C. (2004). Social roles theory of sex differences and similarities: Implications for the partner preferences of women and men. En A. Eagly, A. Beall $y$ R. J. Sternberg (eds.). The psychology of gender (pp. 269-295). New York: Guilford Press.
Edmunds, D., Slayter, B. y Rocheleau, D. (1995). Gendered resource mapping: Focusing on women's spaces in the landscape. Cultural Survival Quarterly, 18(4), 62-68.

Entrena, F. (2008). Globalización, identidad social y hábitos alimentarios. Revista de Ciencias Sociales (Cr), 1(119), 27-38.

Organización de las Naciones Unidad para la Agricultura y la Alimentación. (2015). Comida, territorio y memoria: Situación alimentaria de los pueblos indígenas colombianos. Proyecto TCP/RLA/3403 "Políticas de Seguridad Alimentaria y Nutricional y Pueblos Indígenas en Colombia”. Colombia: Food and Agriculture Organization (FAO).

Gatley, A. (2016). The significance of culinary cultures to diet. British Food Journal, 118(1), 40-59. Doi: http://dx.doi.org/10.1108/BFJ06-2015-0228

González, B. (2019). La preservación de los alimentos tradicionales como elemento formador de la identidad gastronómica de la comunidad otomí de San Pablo Autopan. (Tesis de Licenciatura). México, Universidad Autónoma del Estado de México.

Hernández, M. (2016). Los alimentos en la vida ritual de los nahuas de San Juan Tetelcingo, Guerrero. Un elemento a considerar dentro del patrimonio biocultural. Dimensión Antropológica, 23(66), 64-86.

Hernández, R. y Mendoza, C. P. (2019). Metodología de la investigación. Las rutas cuantitativa, cualitativa y mixta. México: Mc Graw Hill.

Hryciuk, R. E. (2019). La Alquimista de los Sabores: Gastronomic heritage, gender, and the tourist imaginary in Mexico. International Latin American Studies Review, (24), 75-100. Doi: https://doi.org/10.36551/2081-1160. 2019.24.75-100

Hwang, S. (2007). Utilizing qualitative data analysis software: A review of Atlas. ti. Social Science Computer Review, 26(4), 519-527. Doi: https:// doi.org/10.1177/0894439307312485 
López, J., Juárez, L. y Medina, F. (2017). Usos y significados contemporáneos de la comida desde la antropología de la alimentación en América Latina y España. Revista de Dialectología y Tradiciones Populares, 71(2), 327-370. Doi: https://doi.org/10.3989/rdtp.2016.02.001

Matta, R. (2019). Mexico's ethnic culinary heritage and cocineras tradicionales (traditional female cooks). Food and Foodways, 27(3), 211-231. Doi: https://doi.org/10. 1080/07409710.2019.1646481

Mintz, S. W. (1999). La comida como un campo de combate ideológico. VIII Congreso de Antropología. Homenaje a la Xeración Nós. Santiago de Compostela, Federación de Asociaciones de Antropología del Estado Español.

Montoya, M. Á. y Sandoval, E. A. (2013). Marginación sociodemográfica de los otomíes del Estado de México. Papeles de Población, 19(78), 259-291.

Ochoa, T. (2013). Alimentación y diferenciación social: El caso de una comunidad en México. Revista Internacional de Ciencias Sociales, 2(1), 9-20.

Ortiz, I. (2015). La transmisión de los roles de género en los cuentos infantiles. (Tesis de Maestría). Universidad del País Vasco.

Pederzini, C. (2008). La Cocina: ¿Destino o Privilegio Femenino? México: Universidad Iberoaméricana.

Ridgeway, C. L. (2011). Framed by gender: How gender inequality persists in the modern world. Reino Unido: Oxford University Press.

Rodríguez, T. D. J., Chávez, M. C., Thomé H. y Miranda, G. (2017). Elaboración y consumo de tortillas como patrimonio cultural de San Pedro del Rosal, México. Región y Sociedad, 29(70), 155-179. Doi: https://doi. org/10.22198/rys.2017.70.a288

Sánchez, M. y Goldsmith, M. (2000). Reflexiones en torno a la identidad étnica y genérica. Estudios sobre las mujeres indígenas en México. Política y Cultura, (14), 61-88.
Weismantel, M. J. (1991). Tasty meals and bitter gifts: Consumption and production in the Ecuadorian Andes. Food and Foodways, 5(1), 79-94. Doi: https://doi.org/10.1080/07409 710.1991.9961992

\section{Entrevistas}

Cayetano, A. (2019). Patrimonio gastronómico y mujeres otomíes en el Estado de México, México: un problema de roles de género. Entrevista 1 [En persona]. San Pablo Autopan, Estado de México, México.

García, J. (2019). Patrimonio gastronómico y mujeres otomíes en el Estado de México, México: un problema de roles de género. Entrevista 2 [En persona]. San Pablo Autopan, Estado de México, México.

García, L. (2019). Patrimonio gastronómico y mujeres otomíes en el Estado de México, México: un problema de roles de género. Entrevista 3 [En persona]. San Pablo Autopan, Estado de México, México.

García, S. E. (2019). Patrimonio gastronómico y mujeres otomíes en el Estado de México, México: un problema de roles de género. Entrevista 4 [En persona]. San Pablo Autopan, Estado de México, México.

Garduño, E. (2019). Patrimonio gastronómico y mujeres otomíes en el Estado de México, México: un problema de roles de género. Entrevista 5 [En persona]. San Pablo Autopan, Estado de México, México.

Gómez, G. (2019). Patrimonio gastronómico y mujeres otomíes en el Estado de México, México: un problema de roles de género. Entrevista 6 [En persona]. San Pablo Autopan, Estado de México, México.

Gónzalez, L. (2019). Patrimonio gastronómico y mujeres otomíes en el Estado de México, México: un problema de roles de género. Entrevista 7 [En persona]. San Pablo Autopan, Estado de México, México. 
Gónzalez, V. J. (2019). Patrimonio gastronómico y mujeres otomíes en el Estado de México, México: un problema de roles de género. Entrevista 8 [En persona]. San Pablo Autopan, Estado de México, México.

Martínez C. (2019). Patrimonio gastronómico y mujeres otomíes en el Estado de México, México: un problema de roles de género. Entrevista 9 [En persona]. San Pablo Autopan, Estado de México, México.

Martínez, J. (2019). Patrimonio gastronómico y mujeres otomíes en el Estado de México, México: un problema de roles de género. Entrevista 10 [En persona]. San Pablo Autopan, Estado de México, México.
Olmos, M. I. (2019). Patrimonio gastronómico y mujeres otomíes en el Estado de México, México: un problema de roles de género. Entrevista 11 [En persona]. San Pablo Autopan, Estado de México, México.

Téllez, P. (2019). Patrimonio gastronómico y mujeres otomíes en el Estado de México, México: un problema de roles de género. Entrevista 12 [En persona]. San Pablo Autopan, Estado de México, México.

\section{Datos de Filiación}

Arlén Sánchez Valdés. Doctora en Estudios del Turismo. Facultad de Turismo y Gastronomía. Universidad Autónoma del Estado de México (UAEM). Sus líneas de investigación se centran en estudios sobre turismo, sustentabilidad, mercadotecnia, equidad de género.

Bibiana Gónzalez Acosta. Licenciada en Gastronomía. Facultad de Turismo y Gastronomía. Universidad Autónoma del Estado de México. Sus líneas de investigación se centran en estudios sobre el patrimonio gastronómico.

Alejandro Delgado Cruz. Maestro en Estudios Turísticos por la Universidad Autónoma del Estado de México (UAEM). Sus líneas de investigación se centran en estudios sobre turismo, desarrollo y sustentabilidad, paradigmas y modelos de desarrollo turístico, organizaciones y calidad de vida desde la complejidad y la sustentabilidad. Así como, la innovación en el turismo y la gastronomía. 